A randomized trial of brief online interventions to facilitate treatment seeking for social anxiety Margaret R. Tobias ${ }^{1 \mathrm{a}}$, Lauren N. Landy ${ }^{1 \mathrm{a}}$, Michael E. Levin ${ }^{\mathrm{b}}$, \& Joanna J. Arch

${ }^{a}$ Department of Psychology and Neuroscience University of Colorado Boulder 345 UCB

Boulder, CO 80309
${ }^{\mathrm{b}}$ Department of Psychology Utah State University 2810 Old Main Hill Logan, UT 84322

${ }^{1}$ These authors contributed equally to this work.

Corresponding author: Joanna J. Arch, Ph.D. Department of Psychology and Neuroscience University of Colorado Boulder 345 UCB Boulder, CO 80309

Joanna.Arch@Colorado.edu

Biographical statement: M.R.T. is a doctoral candidate in clinical psychology and neuroscience at the University of Colorado Boulder. L.N.L. is a doctoral graduate of the clinical psychology program at the University of Colorado Boulder. M.E.L. is a Professor in the clinical/counseling program at Utah State University. J.J.A. is an Associate Professor in the Department of Psychology and Neuroscience at the University of Colorado Boulder.

Declarations of interest: none.

The authors received no funding from an external source.

Revised submission to:

Behavior Modification

June 9th, 2021 


\begin{abstract}
Objective: This study developed and evaluated a brief, single-session online intervention designed to facilitate treatment seeking among adults with clinically significant social anxiety (SA) symptoms, who generally seek treatment at exceptionally low rates.

Method: Adults $(N=267)$ reporting significant SA symptoms were recruited online and randomized to a brief, single-session online intervention: Education consisted of brief psychoeducation and treatment resources, or Education+Motivation which added treatment seeking-focused motivational content adapted from Motivational Interviewing and Acceptance and Commitment Therapy. Attitudes, intentions, perceived control, and treatment seeking were assessed at Pre, Post, and one-month follow-up (FU).

Results: Both interventions were feasible (90\% completion) and improved all outcomes. At FU, $70 \%$ reported engaging in 1 or more SA treatment-seeking behaviors. Education+Motivation was more effective than Education at improving treatment-seeking attitudes and behaviors. Conclusions: A brief online intervention with educational and motivational content is a promising direction for promoting treatment seeking for adults with SA symptoms.
\end{abstract}

Keywords: social anxiety disorder; internet; values; motivation; acceptance and commitment therapy; motivational interviewing 


\section{A Randomized Trial of Brief Online Interventions to Facilitate Treatment Seeking for Social Anxiety}

Social Anxiety Disorder (SAD) represents one of the most prevalent psychological disorders, with US lifetime prevalence estimates ranging from 5-12\% (Grant et al., 2005; Kessler et al., 2005). SAD often causes substantial distress, impairment, and diminished quality of life (Stein \& Stein, 2008), even at subthreshold levels (Fehm, Beesdo, Jacobi, \& Fiedler, 2008). The disorder typically onsets during childhood or adolescence (Grant et al., 2005), runs a chronic course if untreated (Bruce et al., 2005), and confers risk for mood (Stein et al, 2001) and substance use disorders (Buckner, Bonn-Miller, Zvolensky, \& Schmidt, 2007), causing negative effects across the lifespan.

Fortunately, effective treatment for SAD is available. Cognitive-behavioral therapy (CBT) and its variants demonstrate strong efficacy (e.g., Craske et al, 2014; Hofmann \& Smits, 2008), as do selective serotonin reuptake inhibitors (SSRIs) (van der Linden, Stein, \& van Balkom, 2000). However, many consider CBT the first-line treatment (Mayo-Wilson et al, 2014) due to lower side effects risk (Hansen et al., 2008) and most patients' preference for psychological over pharmacological treatment (McHugh, Whitton, Peckham, Welge, \& Otto, 2013).

Despite the existence of effective treatment, most affected adults never seek or receive any SAD treatment (Keller, 2006). The minority who do seek treatment for SAD wait a median of 16 years after disorder onset (Wang et al., 2005a) and only 38\% of adults receiving past-year SAD treatment received a minimally adequate dose or duration of treatment (Wang et al, 2005b). Furthermore, few adults with anxiety disorders receive CBT, particularly CBT that includes exposure (Wolitzky-Taylor, Zimmerman, Arch, De Guzman, \& Lagomasino, 2015), which is 
generally considered the most effective component of CBT for anxiety disorders (see Glenn et al., 2013). In summary, most adults with SAD do not receive any treatment for their disorder, much less gold-standard evidence-based psychotherapy.

We highlight three key facets underlying SAD’s particularly low rates of help-seeking, with the first two reflecting issues of mental health literacy (MHL; Jorm et al., 1997). When low, MHL impedes mental health treatment seeking (Jorm, 2012). First, there are both general and SAD-specific barriers to seeking treatment (Chartier-Otis, Perreault, \& Bélanger, 2010; Griffiths, 2013; Olfson et al., 2000). Prominent SAD-specific barriers include poorer recognition of SAD as a mental disorder than other anxiety or mood disorders (Coles, Schubert, Heimberg, \& Weiss, 2014), and viewing SAD as an untreatable part of one's personality (see Ruscio et al., 2008). Low recognition of SAD is problematic because failing to identify a mental disorder where one exists generally results in lower treatment seeking (Jorm, 2012). A critical second facet is knowledge of the existence of effective treatments, including exposure-based CBT. Many community adults have not heard of exposure therapy, and providing brief online education about it vastly increases their willingness to try it (Arch, Twohig, Deacon, Landy, \& Bluett, 2015). Taken together, we argue that increasing MHL (i.e., disorder recognition and treatment education) is crucial for increasing SAD treatment seeking behavior.

Additionally, we posit that values-based motivation (e.g., motivation based on who and what one most cares about) may represent a third facet of help-seeking behavior in SAD, in that symptoms of SAD often negatively impact domains that are personally valued, such as personal relationships, education, and work. Clarifying values and leveraging them to increase motivation and commitment to align behavior with one's values is a central focus of both Acceptance and Commitment Therapy (ACT; Hayes, Strosahl, \& Wilson, 1999) and Motivational Interviewing 
(MI; Miller \& Rollnick, 2002). Motivation may be particularly critical for treatment seeking in SAD because the very symptoms of SAD (e.g., fear of talking with strangers or authority figures, initiating or maintaining conversations, or revealing one's personality) present barriers to seeking treatment (Brown \& Barlow, 2014; Moscovitch, 2009). Strong, personally relevant motivation may therefore be needed to overcome these barriers - as has been shown in the parallel literature on treatment seeking in substance use disorders (Lundahl \& Burke, 2009). Further support for use of a motivational enhancement approach to increase treatment seeking arises from recent studies showing that briefly affirming values led to more positive beliefs and intentions about seeking counseling (Lannin,Vogel, \& Heath, 2017; Seidman et al, 2018). Likewise, motivational enhancement was indicated as a frequent component of interventions that improved youths and adolescents' expectations of treatment (Becker, Boustani, Gellatly, \& Chorpita, 2018). Thus, converging evidence across developmental stages suggests that motivational enhancement techniques are well-suited for the task of improving socially anxious adults' beliefs about and intentions toward seeking treatment.

A review of randomized clinical trials (RCTs) that target treatment-seeking in general suggests that psychoeducation and personalized feedback alone are usually insufficient to impact treatment seeking behavior (Gulliver, Griffiths, Christensen, \& Brewer, 2012). Enhancing motivation to seek treatment holds promise for increasing the potency of such interventions. Although ACT has not been used to enhance motivation for anxiety disorder treatment, it has been successfully used to increase motivation and behavioral compliance with a challenging medical rehabilitation treatment that suffers from very low compliance rates (Nelson et al., 2019). Motivational enhancement may also have lasting effects on anxiety-related treatment behavior, supported by the finding that MI before treatment facilitated subsequent CBT 
engagement and outcomes for anxiety disorders (Westra, Arkowitz, \& Dozois, 2009; Westra \& Dozois, 2006). In terms of socially anxious adults that have not yet sought treatment, a small randomized controlled trial $(\mathrm{RCT})(N=27)$ by Buckner and Schmidt (2009) showed that participants who received Motivational Enhancement Therapy (MET; Miller, Zweben, DiClemente, \& Rychtarik, 1992), a three-session, face-to-face intervention based on MI (Miller \& Rollnick, 2002), were significantly more likely to attend a CBT appointment by follow-up compared to those who received brief personalized SAD feedback and psychoeducation. MET also more robustly increased participants' confidence that they could change their anxiety-related behavior, their willingness to attend CBT, and their interest in being contacted by a therapist. However, the very small sample size and need for intensive resources (3.5 hours of individually delivered MET) indicate a need for larger studies evaluating more scalable interventions. Further, a recent large $(N=186)$ RCT comparing pretreatment MI (prior to CBT) versus supportive counseling for adults presenting at a clinic for SAD treatment showed mixed results (Peters et al., 2019). MI led to increased self-reported CBT homework completion, and for participants with higher functional impairment, superior improvements to SAD symptoms. However, session attendance and clinician-rated homework completion did not differ. Further, there was some evidence that MI has a detrimental impact on self-reported SAD symptom severity for those already high in change readiness. Again, both treatments were delivered in three one-hour individual sessions. Thus, more work is needed to develop scalable motivational intervention delivery, assess treatment seeking outcomes (as opposed to treatment outcomes from those already enrolled in or seeking treatment), and reach patients who are not already presenting for treatment.

Online interventions represent a promising format for interventions promoting treatment 
seeking in SAD, as they are scalable and can reach individuals who have not yet presented for treatment. An online format may help reduce the SAD-specific barrier of fear of direct contact with strangers or authority figures (e.g., therapists). Moreover, online interventions may reach individuals with SAD "where they are", as SAD symptoms correlate with a preference for online interaction (Caplan, 2007) and individuals with elevated social anxiety symptoms are overrepresented on online platforms such as Mechanical Turk (MTurk; Shapiro, Chandler, \& Mueller, 2013). To date, one small known trial has tested an online intervention to increase treatment seeking in SAD (Griffiths, Walker, \& Batterham, 2017). They found that an online session of psychoeducation plus one MI exercise, compared to an attention-matched control, improved attitudes and perceived need for, but not intentions for, seeking SAD treatment. As the study did not follow participants post-intervention, however, it could not assess treatmentseeking behavior. Thus, online psychoeducation with minimal motivational content for SAD appears to be helpful for changing treatment seeking attitudes but not intentions, and impact on actual treatment seeking behavior has not been tested. More robust motivational content may be needed to create enduring effects that impact behavior.

\section{The Current Study}

There thus remains a dearth of knowledge regarding how best to improve treatment seeking knowledge, motivations, and especially behavior for socially anxious individuals. We aimed to address this gap by developing, refining, and evaluating in an RCT, two brief, online interventions for improving these outcomes among adults reporting clinically significant social anxiety symptoms. This online approach is well-matched to socially anxious individuals' preferences and behavior (Caplan, 2007; Shapiro et al., 2013) and the current circumstance of global quarantine and social distancing measures due to COVID-19. 
The interventions addressed two barriers to SAD treatment seeking: (1) the gap in MHL (i.e., awareness of SAD as a psychological disorder and knowledge that effective treatment exists), and (2) the gap in understanding how to change SAD treatment seeking attitudes, perceived behavioral control/confidence in one's ability to pursue treatment, behavioral intentions, and actual treatment-seeking behaviors. Our approach in examining these outcomes is informed by a widely used model of behavior change, the Theory of Planned Behavior ${ }^{1}$ (TPB; Ajzen, 1991).

We thus developed and refined two single-session online interventions targeting MHL and MHL + motivation respectively: (1) Education, consisting of brief psychoeducation about SAD symptoms and CBT for SAD, along with information and guidance for locating treatment resources, and (2) Education+Motivation, consisting of Education followed by a brief motivation intervention within the same session. We aimed to examine the effect of the same psychoeducation content with and without additional motivational enhancement content. Importantly, we examined impacts on actual treatment seeking behavior as well as on motivation, in contrast to substantial prior work examining only treatment motivation or readiness. Motivation content was adapted from two traditions with empirical support for facilitating behavior change in a manner that emphasizes personally meaningful values and goals: MI/MET (Miller \& Rollnick, 2002; Lundahl and Burke, 2009) and ACT (Hayes, Strosahl, \& Wilson, 1999). Given brief online delivery, we did not aim to deliver full MI/MET or ACT, but rather to design online motivational exercises based on MI/MET and ACT principles.

\footnotetext{
${ }^{1}$ We considered basing our intervention on another such model of behavior change, the Transtheoretical Model (TTM)/Stages of Change Model (Prochaska \& DiClemente, 1983). However, research has challenged the validity of sequential movement through the TTM stages (e.g., Littell and Girvin, 2002), including relevant previous studies (Buckner and Schmidt, 2009; Maltby and Tolin's, 2007) that found group differences in treatment-seeking behavior in the absence of differences in TTM stages. We thus focused on the TPB as a better-matched model of behavior change within the current context.
} 
This randomized trial evaluated the following a priori indices of feasibility and acceptability: (a) ability to recruit online the targeted sample size of socially anxious adults for the trial (see Power Analysis) and retain at least 70\% through 1-month follow-up (FU), (b) have the majority of participants attend to content and adequately complete the online interventions, and (c) have participants report at least moderate satisfaction with the interventions (operationalized as a mean of 2.83 on the modified Client Satisfaction Questionnaire- 8 at Post). ${ }^{2}$ The clinical trial also evaluated the following efficacy hypotheses: (a) both interventions would improve treatment seeking attitudes, behavioral intentions, and perceived behavioral control from Pre to Post and with gains maintained at FU, but Education+Motivation would lead to greater improvement than Education; and (b) Education+Motivation would improve SAD treatment seeking behavior by FU more than Education alone, though this was exploratory as the study was not fully powered to detect change in behavior.

\section{Method}

\section{Participants}

We recruited a U.S. sample of socially anxious adults $(N=267)$ online through Amazon Mechanical Turk (MTurk) between June and August 2016. A brief description of our study, expected duration, and study payment were presented alongside other MTurk tasks and studies (see Supplemental Materials for additional MTurk recruitment and compensation details). Chandler and Shapiro (2016), including empirical work by Shapiro and colleagues (2013), demonstrate that MTurk-recruited research study participants are similarly honest, attentive, and

\footnotetext{
2 See Method for details. Mean of 2.83 or higher corresponds to a score indicating that neutral or positively valenced choices were selected on all items (note: only one item offered a neutral choice; five offered only negative or positive).
} 
reliable compared to non-MTurk samples, and that MTurk is particularly well suited to reaching populations with social anxiety symptoms.

Of these participants, 195 completed the one-month FU. Eligibility criteria included (1) high social anxiety, per scoring >30 on the Social Phobia Inventory (SPIN; Connor et al., 2000), (2) age 18 or older, (3) fluent in English, (4) not currently enrolled in CBT, bibliotherapy, or online treatment for social anxiety, (5) did not indicate that they would "never consider" treatment for social anxiety on a screener (see below), and (6) on MTurk: had a 95\% or greater HIT approval rate, had not attempted to do the study previously (enforced using TurkGate), and were listed as U.S. residents. Participants resided in diverse locations across the U.S. (see Supplemental Figure 1) and ranged in age from 18 to 66 (see Table 1 for additional sociodemographic and baseline clinical characteristics). Figure 1 presents a CONSORT diagram. Design

This study was an RCT comparing two single-session online interventions: Education versus Education+Motivation. Outcomes were assessed at Pre (prior to randomization), Post (immediately following the intervention) and FU (one month after Post). The study was approved by the University of Colorado Boulder Institutional Review Board and all participants provided informed consent. The study was retroactively registered on ClinicalTrials.gov (identifier NCT04196296) and early documentation of study hypotheses is available upon request in the form of a dissertation proposal that was presented and defended to the dissertation committee. 


\section{Procedure}

\section{Recruitment and Screening}

Interested MTurk participants completed a screening consent followed by a brief screening survey (below). Eligible participants were provided with study details and invited to voluntarily provide informed consent; once consented, they completed a demographic survey. Participants began the study one week later and at that point were randomized 1:1 to condition, allowing for 1) re-administration of the SPIN to assure that scores remained above cutoff, and 2) minimization of post-randomization attrition in MTurk, per recommendation of experts in MTurk research (“Assessing Attrition Bias”, 2013; J. Chandler, personal communication, May 2016). Participation was discontinued for individuals whose SPIN scores did not remain above eligibility threshold at re-administration $(n=65$; Figure 1$)$. There were no significant differences on sociodemographic variables between participants who began the second screen $(n=334)$ and those who did not respond to the invitation for the second screen $(n=85)$ (see Supplemental Materials for full statistical reporting).

\section{Online Intervention Session}

The interventions were implemented using Qualtrics. Exercises were highly interactive (e.g., write-in responses, drag and sort, rank order) both to increase engagement and as an attention/manipulation check. Participants in both conditions were first informed that their responses indicated clinically significant social anxiety symptoms. At the end of both interventions, all participants received identical treatment referral information (see Referral Information). The following subsections describe the intervening content by condition.

Education. Education included descriptions of social anxiety symptoms/SAD and CBT for the treatment of social anxiety, interactive exercises to reflect on symptoms and promote 
engagement, and referrals to various forms of CBT treatment (see Referral Information). Median time to complete the Education condition was 37 minutes $(M=41, S D=16 \text { minutes })^{3}$.

Education+Motivation. In addition to the same Education content, this condition included interactive MI/MET- and ACT-style exercises that drew on participants' personal reflections and experiences. Exercises included reflecting on personal values and how untreated SAD symptoms intruded on living aligned with those values, considering benefits and barriers to seeking treatment, and weighing the roles of short- versus long-term challenges and goals in considering treatment seeking. MI/MET and ACT exercises were adapted from previous interventions (see Supplemental Materials). Intervention content was further refined according to feedback from participants in two rounds of pilot testing on MTurk (see Supplemental Materials for details). The median Education + Motivation condition duration was 69 minutes $(M=77, S D$ $=30$ minutes $)^{4}$. Additional information regarding intervention content can be found in Supplemental Table 1.

Referral Information. At the end of both interventions, participants received identical referral information for evidence-based treatment, which included general resources and specific recommendations for finding in-person, book-based, and online CBT treatments appropriate for participants living in locations throughout the U.S., national mental health emergency hotlines, and brief guidance (e.g., "what to expect"). Attention to referral information was enforced by a required minimum time duration spent on the page (determined per pilot testing, below) before

\footnotetext{
3 There were two participants whose intervention duration exceeded 200 minutes, indicating a high likelihood that the intervention session was not completed in a single sitting (i.e., without a same-day break). We therefore excluded these participants from the duration descriptive statistics but included them in all subsequent analyses, as completion with break did not violate study procedures.

4 See previous footnote. Six participants were excluded from the Education+Motivation duration descriptive statistics (only) due to durations exceeding 200 minutes.
} 
being able to advance to the next page, and self-report (via checkbox: "I certify, honestly, that I have read this page").

\section{Follow-Up Session}

One month after Post, all participants who completed the online intervention session were sent a message through MTurk inviting them to participate in the FU session within the next week. Up to three additional messages were sent within the week to non-responders. The FU session lasted approximately 20 to 30 minutes and consisted of re-administering the outcome measures.

Timing of participant flow through the study is depicted in Supplemental Figure 2. Direct contact between study personnel and participants occurred only in rare cases when answering logistical questions (e.g., clarifying how to access study links) via email.

\section{Measures}

\section{Screener and Basic Clinical Characteristics}

At Pre, the 17-item SPIN (Connor et al., 2000) assessed social anxiety symptoms including fear, avoidance, and physiological symptoms, baseline $\alpha=.82$. A cutoff of 19 has been previously shown to distinguish individuals with SAD from non-clinical and clinical non-SAD samples (Connor et al., 2000). We used a conservative eligibility cutoff of 30+ based on prior SAD research (e.g., Moscovitch, Rodebaugh, \& Hesch, 2012; Moser, Hajcak, Huppert, Foa, \& Simons, 2008). To characterize the sample more fully, participants also completed the following two well-accepted measures of broad anxiety and depression symptoms (respectively) at Pre: The Overall Anxiety Severity and Impairment Scale (OASIS; Norman, Hami Cissell, MeansChristensen, \& Stein, 2006), Pre $\alpha=.62$; and The Patient Health Questionnaire-9 (PHQ-9; Kroenke, Spitzer, \& Williams, 2001), Pre $\alpha=.86$. The order of Pre measures was randomized to 
reduce order effects.

\section{Feasibility and Acceptability Measures}

In addition to recruitment and retention rates, we tracked attention to intervention content via attention checks and careful visual examination of written responses. We also employed the Client Satisfaction Questionnaire (CSQ-8; Attkisson \& Zwick, 1982) at Post $(\alpha=.89)$ to assess acceptability.

\section{Motivation for Treatment}

No established measures based on the TPB have been developed for mental health treatment seeking. We thus built on Kwan and Bryan's (2010) application of the TPB (Ajzen, 1991) to measure a specific domain of behavior, as well as common approaches for assessing TPB variables of attitudes, perceived behavioral control, and behavioral intentions (Fishbein \& Ajzen, 2010), to measure these variables in the context of SAD treatment seeking. We also followed recommendations for assessing complex behaviors such as treatment seeking (A. Bryan, personal communication, April 2016; Griffiths, 2013) by including in the intentions and perceived behavioral control scales each preparatory "step" of the treatment seeking process rather than simply "seeking treatment" (specific steps were drawn from Coles, Turk, Jindra, and Heimberg (2004)'s identification of "critical points" in the "path to initiation of treatment" for social anxiety (see Supplemental Materials for items). Thus, we thoughtfully built upon both previous measurement approaches for adapting TPB to a specific domain and expert recommendations for assessing the behaviors involved in treatment seeking.

Attitudes. Participants evaluated their attitudes toward seeking treatment for social anxiety according to seven semantic differential scale items (e.g., "harmful"/"beneficial"). The 
complete scale is available in the Supplemental Materials. All responses were combined to compute a total score for use in analyses. Cronbach's $\alpha$ 's $=.88-.91$ across timepoints.

Intentions. Participants rated their degree of intention to seek treatment for social anxiety within the next month on one to seven Likert scales. Items assessed intentions for each recommended treatment modality (in-person therapy, bibliotherapy, online). In addition to the items assessing each preparatory step in the treatment seeking process (see above), we included one general item and one item assessing medication, resulting in 13 total intentions items (see Supplemental Materials). Medication was included because it is another evidence-based treatment for social anxiety. However, it was not included in the total score as it was not specifically recommended by the intervention. For the total score (in-person therapy, bibliotherapy, and online therapy) Cronbach's $\alpha$ 's $=.94-.96$ across timepoints.

Perceived Behavioral Control. Participants rated their degree of perceived ability to engage in treatment seeking for social anxiety within the next month with 14 items on a oneseven Likert scale (see Supplemental Materials). Items paralleled the behavioral intentions items (i.e., each step within each treatment modality assessed separately) plus three "general" items to assess perceived behavioral control in the face of barriers, which is theoretically relevant to ACT principles. Cronbach's $\alpha$ 's $=.94-.95$ across timepoints.

\section{Behavior}

We adapted previously established questionnaire items from Buckner and Schmidt (2009) to assess treatment seeking since the intervention (see Supplemental Materials for items), resulting in a checklist of 10 sequential treatment seeking "steps" (listed in Supplemental Table 2). Behavior was assessed at FU to allow time for treatment seeking to occur. Behavioral outcomes included the grand total and within-treatment category sums of steps taken. 


\section{Exploratory Outcomes}

Those who took any steps toward seeking treatment were asked when they took the first step toward each endorsed category (i.e., same day as the online intervention, within two days of the intervention, within $[1,2,3$, or 4$]$ week(s) of the intervention), and whether the treatment was “CBT or related" (“yes," "no," or "unsure”).

\section{Data Processing and Statistical Approach}

Prior to analysis, participant data was attention-checked via visual examination of written responses and systematic examination of self-report questionnaire data. Per Figure 1, six participants were removed for blatantly inappropriate responses to open-ended questions (e.g., responding "I'm bored" to a question about social anxiety). One participant was removed for content-invariant responding on questionnaires.

Analyses were performed using R and SPSS. To evaluate condition differences in demographic and clinical measures at Pre, we used univariate ANOVAs for continuous variables, chi-squared tests for categorical variables and Wilcoxon-Mann Whitney tests for ordinal variables. Chi-squared tests examined condition differences in attrition at each study step following randomization and logistic regression examined whether client satisfaction with the interventions predicted likelihood of attrition. Linear regressions tested condition differences in intervention satisfaction and treatment seeking behavior at FU.

To analyze treatment seeking motivation outcomes (attitudes, intentions, and perceived behavioral control), we constructed multilevel models evaluating condition differences over time in outcomes (e.g., condition by time interactions in predicting outcomes) using the R lmerTest package (Kuznetsova, Brockhoff, \& Christensen, 2017). Predictors included the main effects of condition and time (Pre, Post, FU) and their interaction. Linear and quadratic effects of time 
were evaluated via contrast coding and random effects were allowed to covary. Two-level multilevel models were used to account for motivation outcomes measurements nested within participants by estimating a random intercept and random slope of time for each participant. These models were also run with orthogonal contrast codes for time that specifically evaluated Pre to Post and Post to FU condition differences in outcomes. Effect sizes were estimated for time by condition interactions according to the method described by Feingold (2009) for multilevel models.

\section{Power Analysis}

The study was powered to detect condition differences in change over three time points in the treatment seeking motivational outcomes (i.e., the condition by time interactions). Although the study assessed treatment seeking behavior at FU, it was not fully powered a priori to detect condition differences in this FU outcome ${ }^{5}$.

As approaches for conducting a priori power analysis for multilevel models remain debated and typically require estimation of numerous parameters (Hox, 2010) that were unknown for the present study, we conducted an a priori power calculation using G*Power (Faul, Erdfelder, Lang, \& Buchner, 2007) by conservatively assuming a repeated-measures analysis of variance design to estimate the necessary sample size. To detect a small-to-medium effect size $(d=.3)$ with a two-tailed significance level of .05 , at $95 \%$ power, a total of 116 participants (58 per group) were required at FU. Prior research on MTurk using multiple timepoints (Chandler \& Shapiro, 2016) suggested that we recruit at least $30 \%$ more participants to account for attrition. We thus conservatively planned to enroll another 50 participants to account for attrition and incomplete or unusable data, resulting in a minimum target sample size of 166

\footnotetext{
5 To detect a medium effect size $(d=.3)$ independent samples group difference with a two-tailed significance level of .05 at $80 \%$ power would require a total of 352 participants (176 per group) at follow-up.
} 
participants beginning the intervention. As clinical samples can accrue quickly on MTurk (Chandler \& Shapiro, 2016), we kept the study open for a set period of time (16 days), during which we permitted the sample size to exceed this minimum. Once the final sample size was known, we conducted post hoc power analyses for the multilevel models using the R package SIMR (Green \& MacLeod, 2016), which are presented in the Supplemental Materials.

\section{Results}

\section{Sample Characteristics}

Table 1 presents the baseline demographic and clinical data for participants who completed the online intervention session. There were no condition differences in age, gender, race/ethnicity, education, income, depression (PHQ-9), overall anxiety (OASIS), or social anxiety symptoms (SPIN), $p s \geq .09$. However, the Education+Motivation condition reported higher household income than the Education condition (Table 1), Wilcoxon-Mann Whitney $U=$ 6207.00, $p=.05, r=.02$. As income can be associated with treatment seeking in anxiety disorders (Wang et al., 2005b), we included it as a covariate in subsequent analyses ${ }^{6}$. To account for SAD symptom severity, we also included Pre SPIN score as a covariate.

\section{Attrition}

As depicted in Figure 1's CONSORT diagram, more participants initiated but did not complete the Education+Motivation intervention (11\%) than the Education intervention (3\%), $\chi^{2}$ $=4.63, p=.03$, Cramer's $V=.12$, likely due to its longer length. However, the groups did not differ in attrition from Post to FU, $\chi^{2}=0.25, p=.62$, Cramer's $V=.02$.

\footnotetext{
${ }^{6}$ We reconducted all analyses without income as well. Between group results remained unchanged for attitudes, perceived behavioral control, and intentions, and remained in the same direction and approached significance $(p=$ .08) for treatment-seeking behavioral steps.
} 


\section{Feasibility and Satisfaction Outcomes}

\section{Feasibility}

The data indicated that it was feasible to use MTurk to recruit and retain participants. Within the 16-day recruitment period, the study's recruitment goal was exceeded. Data quality was high; only seven participants were removed due to inadequate or inappropriate responding (per extensive visual inspection of responses, see above). We retained $73 \%$ of participants from randomization through FU (see Figure 1), approximating the $\sim 70 \%$ retention rate in other MTurk psychology research (see Chandler \& Shapiro, 2016). Post-randomization attrition was minimized by the additional eligibility assessment session (see Method), as $65 \%$ occurred prior to randomization. As noted earlier (see Recruitment and Screening), attrition between the first and second screening was not predicted by sociodemographic characteristics.

\section{Satisfaction}

Participants were generally satisfied with the intervention, as assessed by the CSQ-8 at Post $(M=3.07, S D=0.46)$ and FU $(M=2.86, S D=0.61)$, with no condition differences at Post, $t(237)=1.11, p=.27, \eta_{\mathrm{p}}{ }^{2}=.005$. Satisfaction at Post did not predict the likelihood of attrition prior to FU, $b=0.31, p=.40, z=0.85$. However, at $\mathrm{FU}$, those in the Education+Motivation condition $(M=2.98, S D=0.55)$ reported greater satisfaction than those in the Education condition $(M=2.73, S D=0.64), t(191)=3.07, p=0.002, \eta_{\mathrm{p}}^{2}=.05$.

\section{Treatment Seeking Outcomes}

\section{Attitudes}

Overall, attitudes toward SAD treatment seeking increased significantly over time from Pre through FU both linearly, $b=0.27, t(229.94)=3.31, p=.001$, and quadratically, $b=0.23$, $t(238.40)=6.93, p<.001$, see Figure 2 . We decomposed the quadratic effect by examining 
change from Pre to Post and Post to FU. Pre to Post differences revealed condition differences for improvement in attitudes, $b=0.48, t(239.00)=9.167, p<.001, d=0.33 \mathrm{CI}_{95 \%}[0.15,0.51]$, such that attitudes improved more in the Education+Motivation (Pre: $M=4.47, S D=1.17$; Post: $M=5.14, S D=1.17), b=0.67, t(239.00)=8.98, p<.001$, than the Education condition (Pre: $M$ $=4.64, S D=1.11$; Post: $M=4.93, S D=1.23), b=0.29, t(239.00)=3.96, p<.001$. Attitudes somewhat decreased from Post to FU overall, $b=-0.21, t(224.53)=-2.87, p=.005, d=-0.18$ CI95\% [-0.30, -0.06], and there was no difference in this decrease between conditions (Education+Motivation FU: $M=4.99, S D=1.18 ;$ Education $\mathrm{FU}: M=4.65, S D=1.27), b=.05$, $t(224.51)=0.34, p=.74$.

\section{Intentions to Seek Treatment}

Per Figure 3, treatment seeking intentions improved over time from Pre through FU, both linearly, $b=0.22, t(219.12)=2.51, p=.01$, and quadratically, $b=0.42, t(230.46)=11.27, p<$ .001. Conditions did not differ in the linear or quadratic change in intentions over time ( $p$ 's $>$ .15) but did show marginally different rates of change from Pre to Post, $b=0.24, t(239.00)=$ $1.88, p=.06, d=0.18 \mathrm{CI}_{95 \%}[0,0.36]$, such that intentions improved more in the Education+Motivation (Pre: $M=2.80, S D=1.39$; Post: $M=3.66, S D=1.50$ ), $b=0.86$, $t(239.00)=9.41, p<.001$, than Education condition (Pre: $M=2.75, S D=1.35$; Post: $M=3.37$, $S D=1.54), b=0.61, t(239.00)=6.85, p<.001$. Intentions significantly decreased from Post to FU across both conditions, $b=-0.51, t(211.82)=-6.70, p<.001, d=-0.34, \mathrm{CI}_{95 \%}[-0.43,-0.24]$, and there was no difference in this decrease between conditions (Education+Motivation FU: $M=$ 3.19, $S D=1.47 ;$ Education FU: $M=2.93, S D=1.51), b=-0.05, t(211.80)=-0.32, p=.75$.

\section{Perceived Behavioral Control}

Per Figure 4, perceived control over SAD treatment-seeking behavior improved over time 
from Pre through FU, both linearly, $b=0.46, t(225.99)=4.91, p<.001$, and quadratically, $b=$ $0.14, t(241.16)=3.44, p<.001$. However, conditions did not significantly differ in the linear or quadratic effect of time ( $p$ 's $>.30$ ). Perceived control improved from Pre to Post, $b=0.44$, $t(239.00)=7.03, p<.001$, but this effect did not differ significantly by condition (Education+Motivation: Pre: $M=4.05, S D=1.46$; Post: $M=4.55, S D=1.46 ;$ Education : Pre: $M=3.98, S D=1.31$; Post: $M=4.35, S D=1.38), b=0.14, t(239.00)=1.10, p=.27, d=0.10$, $\mathrm{CI}_{95 \%}[-0.08,0.28]$. There was no significant change in perceived control from Post to FU $($ Education + Motivation FU: $M=4.63, S D=1.46 ;$ Education $\mathrm{FU}: M=4.33, S D=1.36), b=$ $0.02, t(227.11)=0.21, p=.83, d=0.01, \mathrm{CI}_{95} \%[-0.11,0.14]$.

\section{Treatment-Seeking Behavior}

Overall, 70\% of participants at FU reported taking at least one step toward seeking treatment for their social anxiety. The median time to taking the first treatment-seeking step was within one week of completing the intervention. At FU, participants in the

Education + Motivation condition took significantly more treatment-seeking steps $(M=2.37, S D$ $=2.15)$ than those in the Education condition $(M=1.84, S D=1.99), F(1,191)=4.13, p=.04$, $\eta_{\mathrm{p}}^{2}=.02 \mathrm{CI} 90 \%=[.00 .07]$. Descriptive statistics for participants' reported steps taken toward different types of treatment-seeking behavior are presented in Supplemental Table 2. Overall, alternative treatments (44.10\% took first step), bibliotherapy (38.46\% took first step), and online treatment (30.77\% took first step) appeared more common, with a smaller portion of participants taking steps toward seeking in-person therapy (17.44\% took first step).

\section{Discussion}

To our knowledge, this study represents the first investigation of an online intervention to facilitate treatment seeking for SAD symptoms and the largest randomized trial to date to 
evaluate a treatment seeking intervention for adults with significant anxiety symptoms of any type. The data supported the study hypotheses regarding online recruitment and intervention feasibility and acceptability. The findings also supported the interventions' initial efficacy at improving treatment-seeking attitudes, intentions, perceived control, and behavior. Finally, the data found partial support for hypotheses regarding differences between conditions, with differences in attitude change toward treatment and actual treatment-seeking behavior favoring the Education+Motivation condition over Education-only.

The online intervention appeared feasible and acceptable. Specifically, we readily recruited a large sample of socially anxious adults and retained $70 \%$ from randomization through FU. The vast majority attended to intervention content, completed their assigned intervention, and reported at least moderate satisfaction at Post.

The intervention efficacy hypotheses were also largely supported, in that treatment seeking attitudes, behavioral intentions, and perceived behavioral control improved from Pre to both Post and FU across conditions. Thus, the interventions succeeded in improving motivation to seek treatment, and these improvements were largely still apparent at FU one month later. Regarding group differences, attitudes toward seeking treatment were improved more by the Education+Motivation condition than by Education alone, although improvement across both conditions declined slightly by one-month FU. Behavioral intentions to seek treatment increased only marginally more through Post by the Education+Motivation condition than the Education condition, and this difference did not endure through FU. Finally, increases in perceived behavioral control did not differ between conditions, possibly indicating that this variable was affected by the provision of practical referral information common across both conditions, 
whereas attitudes and to a lesser extent behavioral intentions were affected by exercises unique to the Education+Motivation condition.

Finally, the interventions and particularly the Education+Motivation intervention successfully increased treatment seeking behavior. Most participants took at least one step toward seeking treatment for social anxiety in the month following completion of the intervention. The exploratory hypothesis was supported in that participants in the Education+Motivation condition took more steps toward treatment seeking than participants in Education alone. This difference reflected a small to medium effect size, and included steps taken toward treatment in general rather than only those specifically recommended by the intervention. Whether the interventions increased CBT-seeking behavior specifically is unknown, as many participants were uncertain whether the treatment they sought was CBT (see Supplemental Table 3). Future research should consider a stronger focus on distinguishing seeking CBT from less evidence-based forms of therapy for SAD, such as general supportive therapy, perhaps by providing more examples of CBT content and strategies. In addition, condition differences in intervention satisfaction favoring the Education+Motivation condition emerged at FU, perhaps because these participants had taken more steps toward treatment.

\section{Comparison to Existing Literature}

In their pilot study $(N=82)$ of psychoeducation plus motivation versus attention control online interventions, Griffiths and colleagues (2017) similarly found that the psychoeducation plus very brief motivation condition led to greater improvement in treatment seeking attitudes and MHL than the attention control condition. However, they did not follow participants beyond Post and thus did not assess treatment-seeking behavior. Intentions to seek treatment for SAD also improved in their study but did not significantly differ by condition - paralleling the current 
study's finding. The present study differs however, in that it was much larger, evaluated participants at FU, and found differences in treatment seeking behavior and marginal postintervention differences in treatment seeking intentions.

This study is consistent with Buckner and Schmidt's study (2009) in that both found that motivational interventions were effective at increasing treatment-seeking for SAD or social anxiety symptoms. Their study was embedded in a clinic and the outcome was attending a CBT appointment in that clinic for SAD. Their intervention's higher intensity, potency, and directedness reflect its multi-session, individual, one-on-one sessions with mental health trainees and use of on-site treatment referrals. The present study thus extends their findings by using a single-session online intervention within a larger online sample, increasing the intervention's reach and scalability. The present study also had better retention at follow-up despite Buckner and Schmidt's substantially higher compensation. These differences are perhaps in part due to the facilitative effect of the online format for socially anxious individuals in particular, which is congruent with Shapiro and colleagues' (2013) finding that adults with clinically noteworthy social anxiety were less comfortable disclosing clinical information in-person than less socially anxious individuals, but no less comfortable disclosing online.

The current study's findings cannot be directly compared to Peters and colleagues' (2019) large RCT $(N=186)$ of MI versus supportive counseling pretreatment for adults presenting at a clinic for CBT treatment of their SAD, given our focus on treatment seeking rather than CBT outcomes and use of a sample not already presenting for SAD treatment. Still, we highlight their findings that MI facilitated SAD symptom improvement amongst those higher in functional impairment but dampened improvement amongst those higher in change readiness. Motivational interventions are perhaps best matched to getting socially anxious individuals to 
seek treatment (per the present study and Buckner \& Schmidt, 2009) and after they have committed to treatment, to individuals with more functional impairment (per Peters et al., 2019).

Our findings also generally align with Gulliver and colleagues' (2012) review of randomized interventions aiming to facilitate mental health help seeking in general samples and Brijnath, Protheroe, Mahtani, and Antoniades's (2016) review of online interventions to improve MHL. However, they found that psychoeducation was insufficient to improve help seeking behavior whereas we found that most participants reported taking at least one step toward treatment seeking even in the Education only condition.

\section{Study Strengths, Limitations, and Future Directions}

The present study's brief, single-session, and online nature enhances its potential for scalability, dissemination, and cost efficacy. Recruiting and intervening online appears to be a good match for socially anxious adults, given their fears of live social interaction but not of online interaction, and their strong presence online, including on MTurk (Caplan et al., 2007; Shapiro et al., 2013). The present study's brevity and lack of contact with providers, though strengths from a scalability perspective, may have nonetheless limited the current intervention's power to impact behavior change. The current use of broader referrals rather than connecting to providers at a specific clinic enhanced applicability across varied U.S. locations (see Supplemental Figure 3) and the ability to seek preferred forms of treatment. Still, future iterations should consider more geographically or personally tailored approaches to treatment referral to simplify the process of seeking in-person therapy.

The two interventions were not matched for length of contact, given our aim to conduct a study examining the respective contribution of treatment seeking motivational enhancement content over and above psychoeducation, which was held constant between the two conditions. 
This trial is similar in design both to dismantling trials as well as to hundreds of others in which both conditions have access to usual care (paralleling psychoeducation in the current trial) but only one is given the intervention of interest (motivational enhancement in the current trial). Like all such trials, condition differences should be interpreted with some caution as the effect of motivational content is confounded with prolonged participation. That stated, the conditions were both matched for being delivered entirely online without provider contact. Further, the current comparison is externally valid, as the Education condition was designed to reflect what a patient might hear from an informed provider about treatment options, thus reflecting usual care for individuals examining potential treatment for social anxiety. Still, future work should unpack the respective contributions of content and length of contact.

Likewise, the lack of a no-intervention control condition limits our ability to conclusively state that treatment seeking behavioral steps were induced by the interventions as opposed to by the passage of time. However, poor recognition of SAD as a disorder and as a treatable condition and low awareness of effective treatments (Coles et al., 2014; Ruscio et al., 2008), very low rates of SAD-focused treatment seeking generally (Iza et al., 2013), and among the minority who seek treatment for SAD, a median of 16 years before treatment (Wang et al., 2005a), renders this explanation unlikely. Contradicting these findings, this explanation would imply that individuals with significant SAD symptoms are indeed aware that their symptoms are treatable and are likely to spontaneously begin seeking treatment despite not seeking treatment prior (a study inclusion criteria). Though unlikely, to definitively rule-out such interpretations, future work in this domain would benefit from including a no-intervention control condition.

Several additional factors limited our study. First, the findings must be contextualized by our recruitment of an incentivized participant sample. However, as noted, research reviewed by 
Chandler and Shapiro (2016), including empirical work by Shapiro and colleagues (2013), illustrates that MTurk workers are comparably honest, attentive, and reliable compared to nonMTurk samples, and that MTurk is well suited to reaching populations with clinically relevant symptoms. Second, we relied on self-reported SAD symptoms for study eligibility, and thus cannot fully generalize our findings to those with SAD based on diagnostic assessment. However, we used a conservative, frequently used cutoff on a measure that correlates well with SAD diagnosis. Third, the study was not fully powered to detect changes in behavior at followup and was not powered to test for group differences in specific treatment modalities (e.g., inperson therapy vs. bibliotherapy vs. alternative treatments). Additionally, despite our large sample size compared to existing literature, post hoc power analyses (see Supplemental Materials) suggested that our multilevel models only achieved 60-72\% power to detect linear (Pre vs. FU) change in outcomes. Thus, larger future studies are warranted. Similarly, the popularity of bibliotherapy and alternative treatments warrants further study, particularly in light of the fact that the data were collected several years ago and the coronavirus pandemic may have further increased many people's openness to online and bibliotherapy treatment options. The cost-effectiveness, flexibility, independence, and lack of requiring in-person contact of bibliotherapy may have appealed to participants. Lastly, the study was only retrospectively registered on ClinicalTrials.gov, as preregistration was not standard practice for internally funded studies at the time of data collection.

This study was in some ways also constrained by limitations in the field, as the literature lacked a single comprehensive measure of motivation for treatment seeking. We drew from existing data and theory by adapting established measures to this specific type of behavior. Similarly, the central theoretical model on which to base treatment seeking interventions is 
underdeveloped (Gulliver et al., 2012), highlighting the need to identify specific modifiable barriers to treatment seeking in SAD.

\section{Summary and Conclusions}

To our knowledge, this is the largest randomized study to date to evaluate the acceptability, feasibility, and initial efficacy of an anxiety-focused treatment seeking intervention (relative to an education-only intervention control) and the first to use online interventions to facilitate treatment seeking for adults with significant social anxiety symptoms. The promising findings align with recent recommendations to employ online modalities and direct-to-consumer strategies to improve treatment dissemination generally (e.g., Gallo, Comer \& Barlow, 2013; Kazdin \& Blase, 2011). This study provides a promising approach for addressing barriers to treatment seeking for socially anxious adults and warrants a larger trial that is powered to detect change in distinct forms of treatment seeking and to clarify the intervention's broader public health potential. 


\section{References}

Ajzen, I. (1991). The theory of planned behavior. Organizational Behavior and Human Decision Processes, 50(2), 179-211.

Arch, J. J., Twohig, M. P., Deacon, B. J., Landy, L. N., \& Bluett, E. J. (2015). The credibility of exposure therapy: Does the theoretical rationale matter? Behaviour Research and Therapy, 72, 81-92.

Assessing attrition bias. (2013). Retrieved from https://ies.ed.gov/ncee/wwc/Docs/ReferenceResources/wwc_attrition_v2.1.pdf.

Attkisson, C. C., \& Zwick, R. (1982). The client satisfaction questionnaire: Psychometric properties and correlations with service utilization and psychotherapy outcome. Evaluation and Program Planning, 5(3), 233-237.

Becker, K. D., Boustani, M., Gellatly, R., \& Chorpita, B. F. (2018). Forty years of engagement research in children's mental health services: Multidimensional measurement and practice elements. Journal of Clinical Child \& Adolescent Psychology, 47(1), 1-23.

Brijnath, B., Protheroe, J., Mahtani, K. R., \& Antoniades, J. (2016). Do web-based mental health literacy interventions improve the mental health literacy of adult consumers? Results from a systematic review. Journal of Medical Internet Research, 18(6), e165.

Brown, T. A., \& Barlow, D. H. (2014). Anxiety and related disorders interview schedule for DSM-5 (ADIS-5L): Lifetime version. Client interview schedule. Oxford University Press.

Bruce, S. E., Yonkers, K. A., Otto, M. W., Eisen, J. L., Weisberg, R. B., Pagano, M., ... \& Keller, M. B. (2005). Influence of psychiatric comorbidity on recovery and recurrence in generalized anxiety disorder, social phobia, and panic disorder: a 12-year prospective study. The American Journal of Psychiatry, 162(6), 1179-1187. 
Buckner, J. D., Bonn-Miller, M. O., Zvolensky, M. J., \& Schmidt, N. B. (2007). Marijuana use motives and social anxiety among marijuana using young adults. Addictive Behaviors, 32(10), 2238-2252.

Buckner, J. D., \& Schmidt, N. B. (2009). A randomized pilot study of motivation enhancement therapy to increase utilization of cognitive-behavioral therapy for social anxiety. Behaviour Research and Therapy, 47(8), 710-715.

Caplan, S. E. (2007). Relations among loneliness, social anxiety, and problematic Internet use. CyberPsychology \& Behavior, 10(2), 234-242.

Chandler, J., \& Shapiro, D. (2016). Conducting clinical research using crowdsourced convenience samples. Annual Review of Clinical Psychology, 12, 53-81.

Chartier-Otis, M., Perreault, M., \& Bélanger, C. (2010). Determinants of barriers to treatment for anxiety disorders. Psychiatric Quarterly, 81(2), 127-138.

Coles, M. E., Schubert, J. R., Heimberg, R. G., \& Weiss, B. D. (2014). Disseminating treatment for anxiety disorders: Step 1: Recognizing the problem as a precursor to seeking help. Journal of Anxiety Disorders, 28(8), 737-740.

Coles, M. E., Turk, C. L., Jindra, L., \& Heimberg, R. G. (2004). The path from initial inquiry to initiation of treatment for social anxiety disorder in an anxiety disorders specialty clinic. Journal of Anxiety Disorders, 18(3), 371-383.

Connor, K. M., Davidson, J. R., Churchill, L. E., Sherwood, A., Foa, E., \& Weisler, R. H. (2000). Psychometric properties of the Social Phobia Inventory (SPIN). New self-rating scale. The British Journal of Psychiatry, 176(4), 379-386.

Craske, M. G., Niles, A. N., Burklund, L. J., Wolitzky-Taylor, K. B., Vilardaga, J. C., Arch, J. J., ... Lieberman, M. D. (2014). Randomized controlled trial of cognitive behavioral therapy 
and acceptance and commitment therapy for social phobia: Outcomes and moderators. Journal of Consulting and Clinical Psychology, 82(6), 1034-1048.

Eifert, G. H., \& Forsyth, J. P. (2005). Acceptance and commitment therapy for anxiety disorders: A practitioner's treatment guide to using mindfulness, acceptance, and values-based behavior change strategies. Oakland, CA: New Harbinger Publications.

Fair Payment (2016, April 4). Retrieved from http://wiki.wearedynamo.org/index.php?title=Fair_payment

Faul, F., Erdfelder, E., Lang, A. G., \& Buchner, A. (2007). G*Power 3: A flexible statistical power analysis program for the social, behavioral, and biomedical sciences. Behavior Research Methods, 39(2), 175-191.

Fehm, L., Beesdo, K., Jacobi, F., \& Fiedler, A. (2008). Social anxiety disorder above and below the diagnostic threshold: Prevalence, comorbidity and impairment in the general population. Social Psychiatry and Psychiatric Epidemiology, 43(4), 257-265.

Feingold, A. (2009). Effect sizes for growth-modeling analysis for controlled clinical trials in the same metric as for classical analysis. Psychological Methods, 14(1), 43-53.

Fishbein, M., \& Ajzen, I. (2011). Predicting and changing behavior: The reasoned action approach. Taylor \& Francis.

Gallo, K. P., Comer, J. S., \& Barlow, D. H. (2013). Direct-to-consumer marketing of psychological treatments for anxiety disorders. Journal of Anxiety Disorders, 27(8), 793801.

Glenn, D., Golinelli, D., Rose, R. D., Roy-Byrne, P., Stein, M. B., Sullivan, G., ... Craske, M. G. (2013). Who gets the most out of cognitive behavioral therapy for anxiety disorders? The 
role of treatment dose and patient engagement. Journal of Consulting and Clinical Psychology, 81(4), 639-649.

Grant, B. F., Hasin, D. S., Blanco, C., Stinson, F. S., Chou, S. P., Goldstein, R. B., ... Huang, B. (2005). The epidemiology of social anxiety disorder in the United States: Results from the National Epidemiologic Survey on Alcohol and Related Conditions. Journal of Clinical Psychiatry, 66(11), 1351-1361.

Green, P., \& MacLeod, C. J. (2016). SIMR: An R package for power analysis of generalized linear mixed models by simulation. Methods in Ecology and Evolution, 7(4), 493-498.

Griffiths, K. M. (2013). Towards a framework for increasing help-seeking for social anxiety disorder. Australian \& New Zealand Journal of Psychiatry, 47(10), 899-903.

Griffiths, K. M., Walker, J., \& Batterham, P. J. (2017). Help seeking for social anxiety: A pilot randomised controlled trial. Digital Health, 3, 1-12.

Gulliver, A., Griffiths, K. M., Christensen, H., \& Brewer, J. L. (2012). A systematic review of help-seeking interventions for depression, anxiety and general psychological distress. BMC Psychiatry, 12(1), 81.

Hansen, R. A., Gaynes, B. N., Gartlehner, G., Moore, C. G., Tiwari, R., \& Lohr, K. N. (2008). Efficacy and tolerability of second-generation antidepressants in social anxiety disorder. International Clinical Psychopharmacology, 23(3), 170-179.

Harris, R. (2009). ACT made simple: An easy-to-read primer on acceptance and commitment therapy. Oakland, CA: New Harbinger Publications.

Hayes, S. C., Strosahl, K. D., \& Wilson, K. G. (1999). Acceptance and commitment therapy: An experiential approach to behavior change. New York, NY: Guilford Press. 
Hofmann, S. G., \& Smits, J. A. (2008). Cognitive-behavioral therapy for adult anxiety disorders: A meta-analysis of randomized placebo-controlled trials. The Journal of Clinical Psychiatry, 69(4), 621-632.

Horton, J. J., \& Chilton, L. B. (2010, June). The labor economics of paid crowdsourcing. Paper presented at the 11th Association for Computing Machinery Conference on Electronic Commerce, Cambridge, MA.

Hox, J. (2010). Sample sizes and power analysis in multilevel regression. In Multilevel analysis: Techniques and applications ( $2^{\text {nd }}$ Ed.) (pp. 233-256). Taylor \& Francis Group.

Iza, M., Olfson, M., Vermes, D., Hoffer, M., Wang, S., \& Blanco, C. (2013). Probability and predictors of first treatment contact for anxiety disorders in the United States: Analysis of data from the National Epidemiologic Survey on Alcohol and Related Conditions (NESARC). The Journal of Clinical Psychiatry, 74(11), 1093-1100.

Jorm, A. F. (2012). Mental health literacy: Empowering the community to take action for better mental health. The American Psychologist, 67(3), 231-243.

Jorm, A. F., Korten, A. E., Jacomb, P. A., Christensen, H., Rodgers, B., \& Pollitt, P. (1997). "Mental health literacy": A survey of the public's ability to recognise mental disorders and their beliefs about the effectiveness of treatment. The Medical Journal of Australia, 166(4), 182-186.

Kazdin, A. E., \& Blase, S. L. (2011). Rebooting psychotherapy research and practice to reduce the burden of mental illness. Perspectives on Psychological Science, 6(1), 21-37.

Keller, M. B. (2006). Social anxiety disorder clinical course and outcome: Review of Harvard/Brown Anxiety Research Project (HARP) findings. The Journal of Clinical Psychiatry, 67(Suppl 12), 14-19. 
Kessler, R. C., Berglund, P., Demler, O., Jin, R., Merikangas, K. R., \& Walters, E. E. (2005). Lifetime prevalence and age-of-onset distributions of DSM-IV disorders in the National Comorbidity Survey Replication. Archives of General Psychiatry, 62(6), 593-602.

Kroenke, K., Spitzer, R. L., \& Williams, J. B. (2001). The PHQ-9: Validity of a brief depression severity measure. Journal of General Internal Medicine, 16(9), 606-613.

Kuznetsova A., Brockhoff, P. B., Christensen, R. H. B. (2017). lmerTest package: Tests in linear mixed effects models. Journal of Statistical Software, 82(13), 1-26.

Kwan, B. M., \& Bryan, A. D. (2010). Affective response to exercise as a component of exercise motivation: Attitudes, norms, self-efficacy, and temporal stability of intentions. Psychology of Sport and Exercise, 11(1), 71-79.

Lannin, D. G., Vogel, D. L., \& Heath, P. J. (2017). Can reflecting on personal values online increase positive beliefs about counseling? Journal of Counseling Psychology, 64(3), 261-268.

Levin, M. E., Haeger, J., Pierce, B. G. \& Twohig, M. P. (2017). Web-based acceptance and commitment therapy for mental health problems in college students: A randomized controlled trial. Behavior Modification, 41(1), 141-162.

Littell, J. H., \& Girvin, H. (2002). Stages of change: A critique. Behavior Modification, 26(2), 223-273.

Lundahl, B., \& Burke, B. L. (2009). The effectiveness and applicability of motivational interviewing: A practice-friendly review of four meta-analyses. Journal of Clinical Psychology, 65(11), 1232-45.

Mayo-Wilson, E., Dias, S., Mavranezouli, I., Kew, K., Clark, D. M., Ades, A. E., \& Pilling, S. (2014). Psychological and pharmacological interventions for social anxiety disorder in 
adults: A systematic review and network meta-analysis. The Lancet Psychiatry, 1(5), 368-376.

McHugh, R. K., Whitton, S. W., Peckham, A. D., Welge, J. A., \& Otto, M. W. (2013). Patient preference for psychological vs. pharmacological treatment of psychiatric disorders: A meta-analytic review. The Journal of Clinical Psychiatry, 74(6), 595-602.

Miller, W. R., \& Rollnick, S. (2002). Motivational interviewing: Preparing people for change. New York, NY: Guilford Press.

Miller, W. R., Zweben, A., DiClemente, C. C., \& Rychtarik, R. G. (1992). Motivational enhancement therapy manual: A clinical tool for therapists treating individuals with alcohol abuse and dependence. Rockville, MD: National Institute on Alcohol Abuse and Alcoholism.

Moscovitch, D. A. (2009). What is the core fear in social phobia? A new model to facilitate individualized case conceptualization and treatment. Cognitive and Behavioral Practice, $16(2), 123-134$.

Moscovitch, D. A., Rodebaugh, T. L., \& Hesch, B. D. (2012). How awkward! Social anxiety and the perceived consequences of social blunders. Behaviour Research and Therapy, 50(2), 142-149.

Moser, J. S., Hajcak, G., Huppert, J. D., Foa, E. B., \& Simons, R. F. (2008). Interpretation bias in social anxiety as detected by event-related brain potentials. Emotion, 8(5), 693-700.

Nelson, C. J., Saracino, R. M., Napolitano, S., Pessin, H., Narus, J. B., \& Mulhall, J. P. (2019). Acceptance and commitment therapy to increase adherence to penile injection therapybased rehabilitation after radical prostatectomy: Pilot randomized controlled trial. The Journal of Sexual Medicine, 16(9), 1398-1408. 
Norman, S. B., Cissell, S. H., Means-Christensen, A. J., \& Stein, M. B. (2006). Development and validation of an overall anxiety severity and impairment scale (OASIS). Depression and Anxiety, 23(4), 245-249.

Olfson, M., Guardino, M., Struening, E., Schneier, F. R., Hellman, F., \& Klein, D. F. (2000). Barriers to the treatment of social anxiety. The American Journal of Psychiatry, 157(4), 521-527.

Peters, L., Romano, M., Byrow, Y., Gregory, B., McLellan, L. F., Brockveld, K., ... \& Rapee, R. M. (2019). Motivational interviewing prior to cognitive behavioural treatment for social anxiety disorder: A randomised controlled trial. Journal of Affective Disorders, 256, 7078.

Prochaska, J. O., \& DiClemente, C. C. (1983). Stages and processes of self-change of smoking: Toward an integrative model of change. Journal of Consulting and Clinical Psychology, 51(3), 390-395.

Ruscio, A. M., Brown, T. A., Chiu, W. T., Sareen, J., Stein, M. B., \& Kessler, R. C. (2008). Social fears and social phobia in the USA: Results from the National Comorbidity Survey Replication. Psychological Medicine, 38(1), 15-28.

Seidman, A. J., Wade, N. G., Lannin, D. G., Heath, P. J., Brenner, R. E., \& Vogel, D. L. (2018). Self-affirming values to increase student veterans' intentions to seek counseling. Journal of Counseling Psychology, 65(5), 653-660.

Shapiro, D. N., Chandler, J., \& Mueller, P. A. (2013). Using Mechanical Turk to study clinical populations. Clinical Psychological Science, 1(2), 213-220. 
Stein, M. B., Fuetsch, M., Müller, N., Höfler, M., Lieb, R., \& Wittchen, H. U. (2001). Social anxiety disorder and the risk of depression: A prospective community study of adolescents and young adults. Archives of General Psychiatry, 58(3), 251-256.

Stein, M. B., \& Stein, D. J. (2008). Social anxiety disorder. The Lancet, 371, 1115-1125.

van der Linden, G. J. H., Stein, D. J, \& van Balkom, A. J. L. M. (2000). The efficacy of the selective serotonin reuptake inhibitors for social anxiety disorder (social phobia): A metaanalysis of randomized controlled trials. International Clinical Psychopharmacology, 15(Supp12), S15-S23.

Wang, P. S., Berglund, P., Olfson, M., Pincus, H. A., Wells, K. B., \& Kessler, R. C. (2005a). Failure and delay in initial treatment contact after first onset of mental disorders in the National Comorbidity Survey Replication. Archives of General Psychiatry,62(6), 603-613.

Wang, P. S., Lane, M., Olfson, M., Pincus, H. A., Wells, K. B., \& Kessler, R. C. (2005b). Twelve-month use of mental health services in the United States: Results from the National Comorbidity Survey Replication. Archives of General Psychiatry, 62(6), 629640.

Webber, K. H., Tate, D. F., \& Quintiliani, L. M. (2008). Motivational interviewing in internet groups: A pilot study for weight loss. Journal of the American Dietetic Association, 108(6), 1029-1032.

Westra, H. A. (2012). Motivational interviewing in the treatment of anxiety. New York, NY: Guilford Press.

Westra, H. A., Arkowitz, H., \& Dozois, D. J. A. (2009). Adding a motivational interviewing pretreatment to cognitive behavioral therapy for generalized anxiety disorder: A preliminary randomized controlled trial. Journal of Anxiety Disorders, 23(8), 1106-1117. 
Westra, H. A., \& Dozois, D. J. A. (2006). Preparing clients for cognitive behavioral therapy: a randomized pilot study of motivational interviewing for anxiety. Cognitive Therapy and Research, 30(4), 481-498.

Wolitzky-Taylor, K., Zimmermann, M., Arch, J. J., De Guzman, E., \& Lagomasino, I. (2015). Has evidence-based psychosocial treatment for anxiety disorders permeated usual care in community mental health settings? Behaviour Research and Therapy, 72, 9-17. 


\section{Table 1}

Post-Intervention Sample Characteristics: Demographic and Baseline Clinical Variables

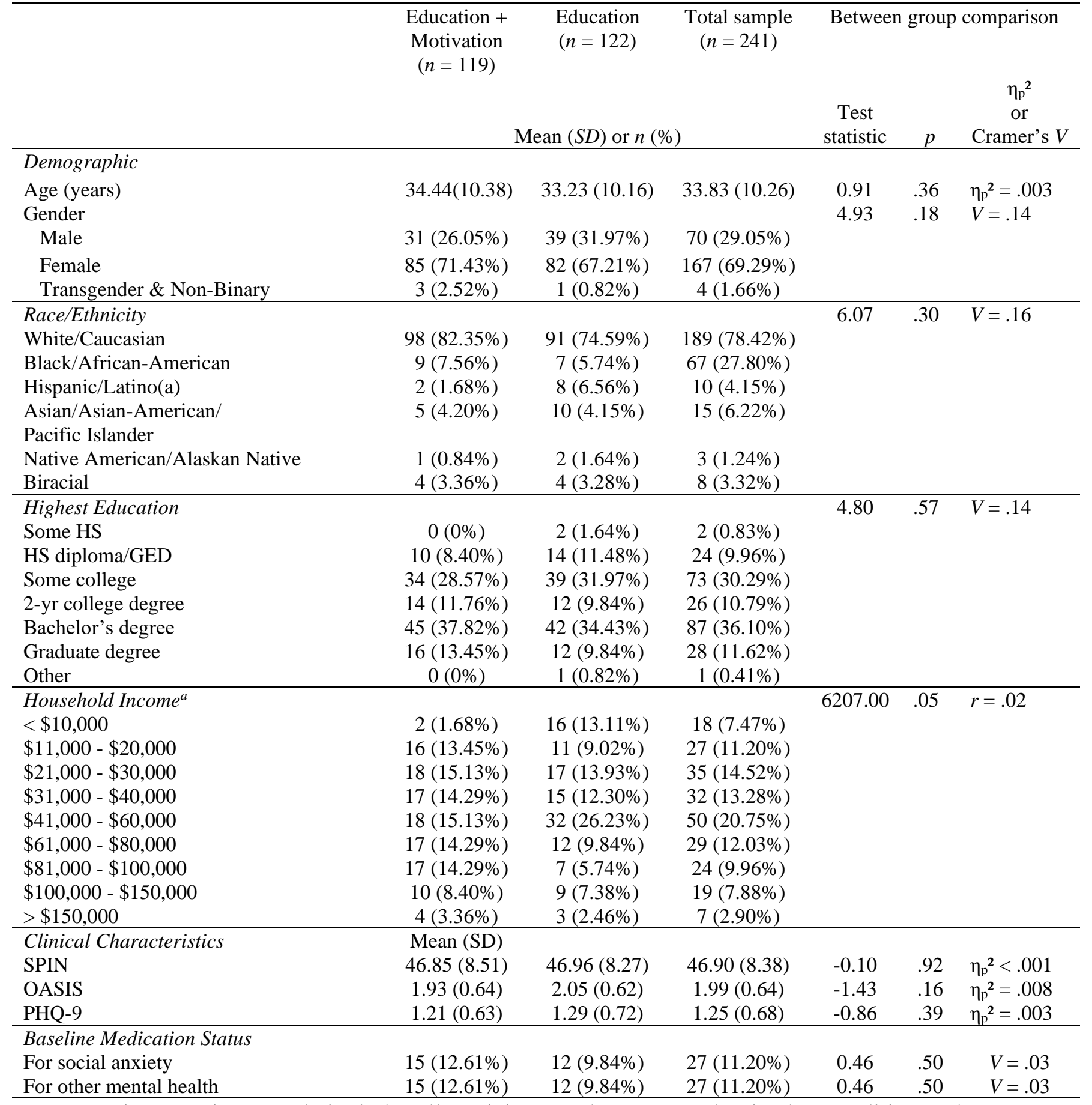

Note. Post-intervention sample includes all participants who were randomized to condition and

successfully completed intervention session. T-tests were used for continuous data, chi square for categorical, U for ordinal. ${ }^{\text {a }}$ Wilcoxon-Mann Whitney test used due to ordinal nature of the variable. 


\section{Figure 1}

CONSORT Diagram Detailing Participant Flow Through Study

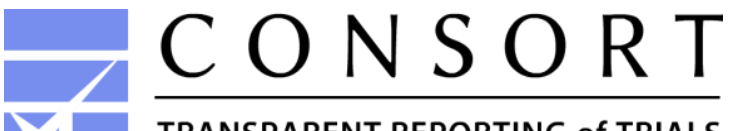

TRANSPARENT REPORTING of TRIALS

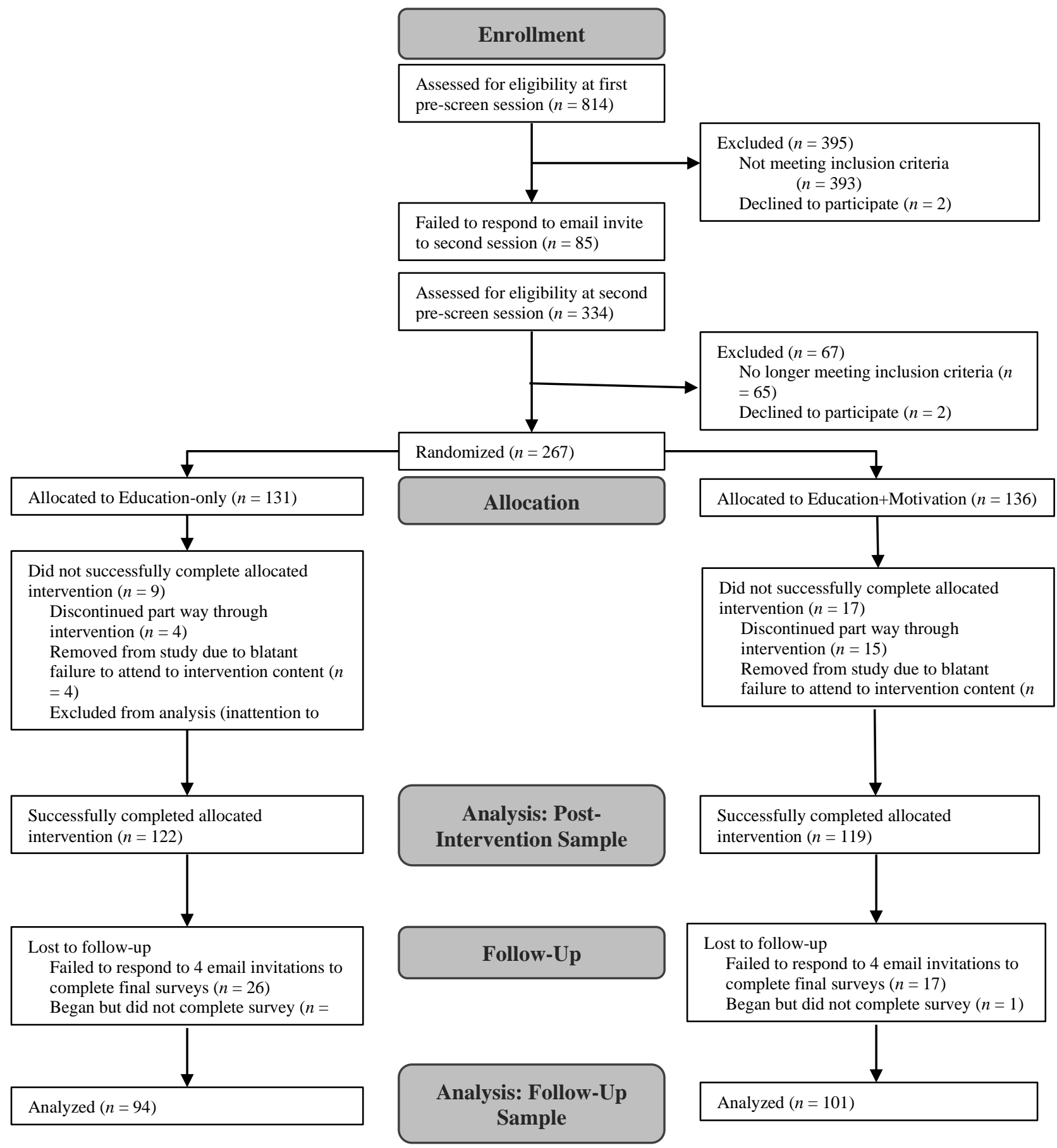




\section{Figure 2}

Attitudes Toward Seeking Treatment for SAD by Condition Over Time.

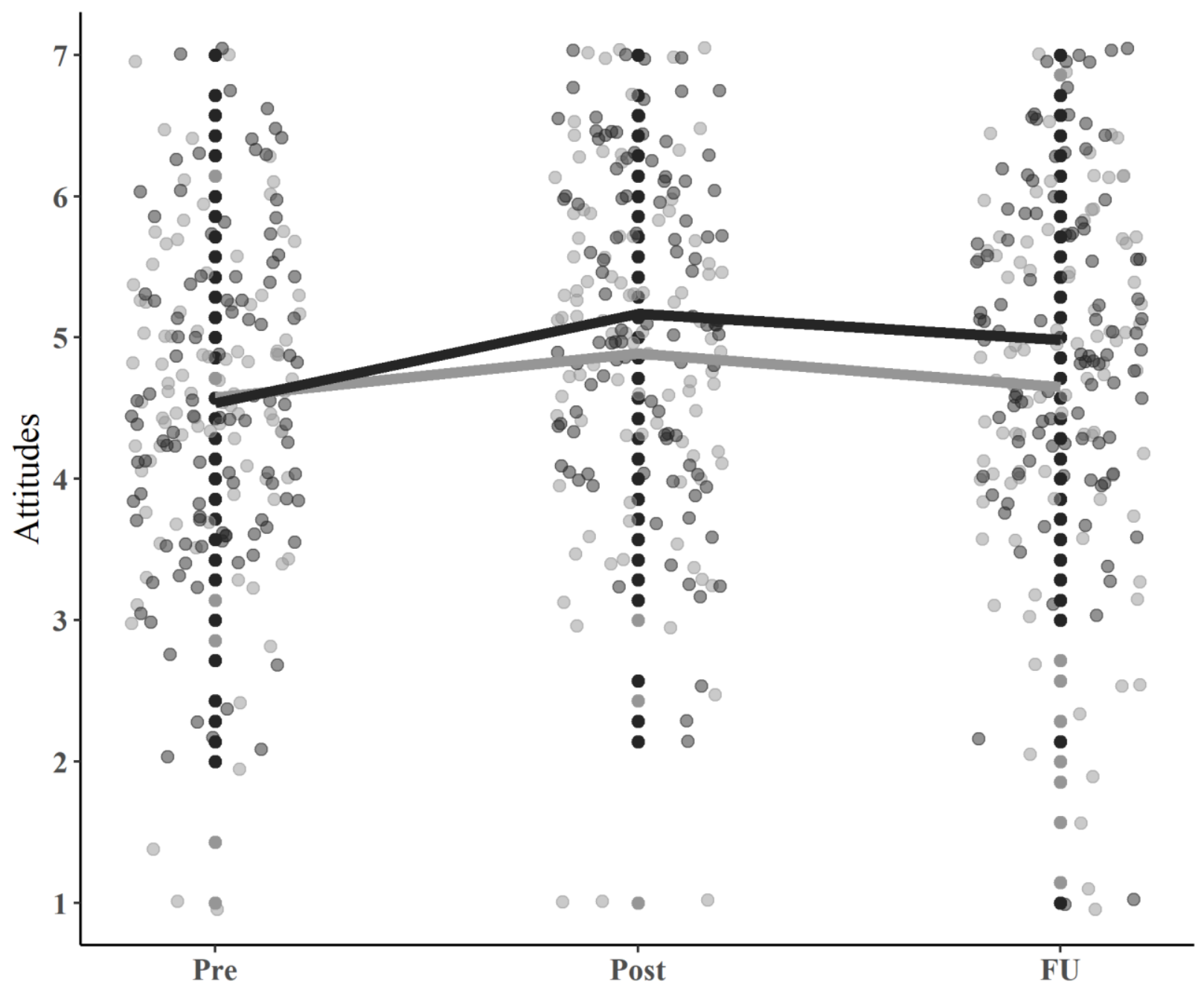

\section{Condition Education Motivation}

Note. Attitudes significantly improved from Pre to FU in the Education+Motivation $(p<.001)$ but not Education $(p=.68)$ treatment condition (see Results). Attitudes were measured using a seven item seven-point semantic differential scale in which higher scores correspond with more favorable attitudes toward seeking treatment for SAD (see Method). 


\section{Figure 3}

Intentions to Seek Treatment for SAD by Condition Over Time

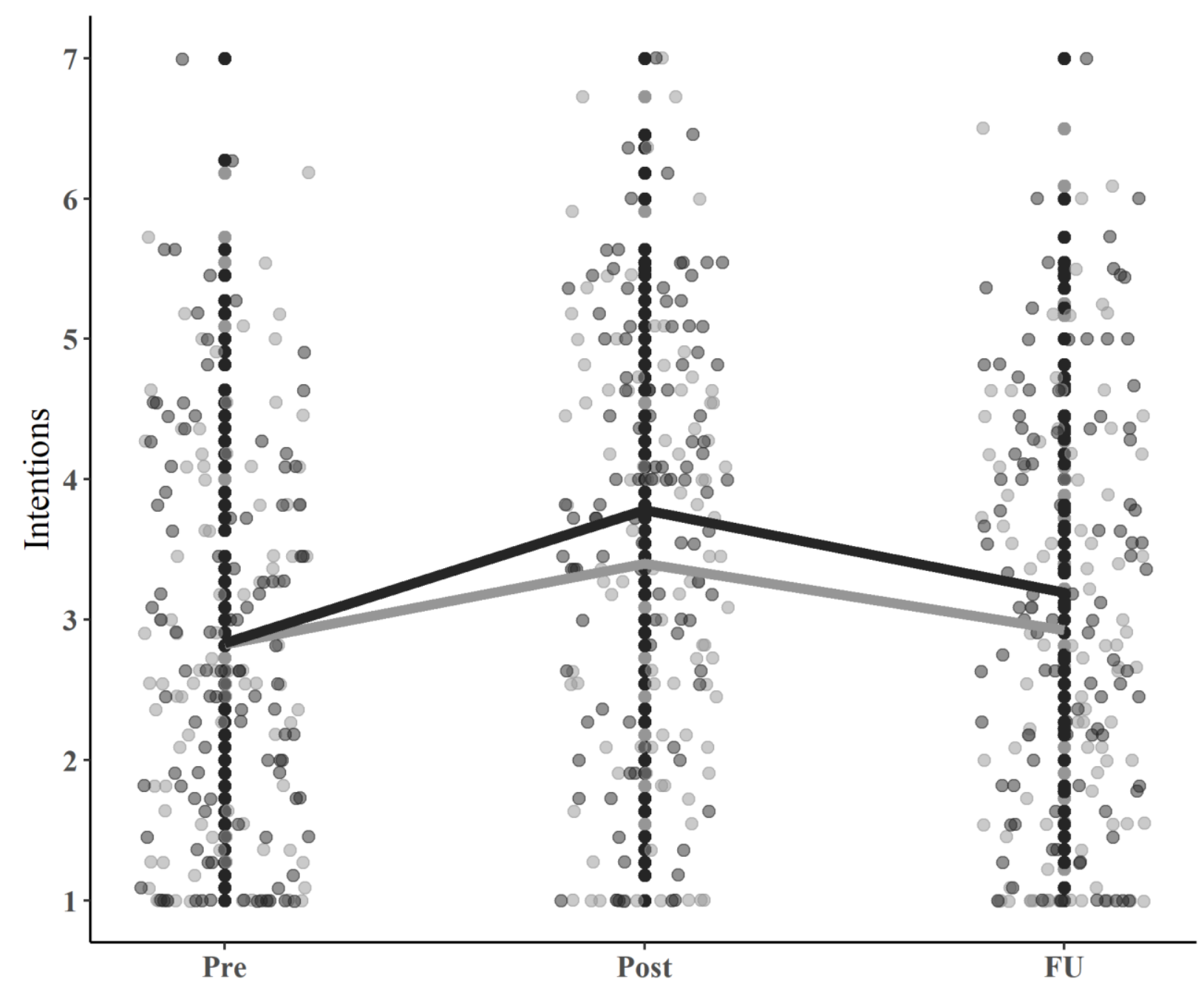

\section{Condition Education}

Note. The Pre-FU improvement in intentions did not significantly differ between treatment groups $(p=.34)$, but the Pre-Post improvement was marginally greater $(p=.06)$ in the Education+Motivation than Education condition. Intentions were measured using a 13 item 1-7 Likert scale measure in which higher scores represent greater intentions to seek treatment for SAD (see Method). 


\section{Figure 4}

Perceived Behavioral Control for Seeking Treatment for SAD by Condition Over Time

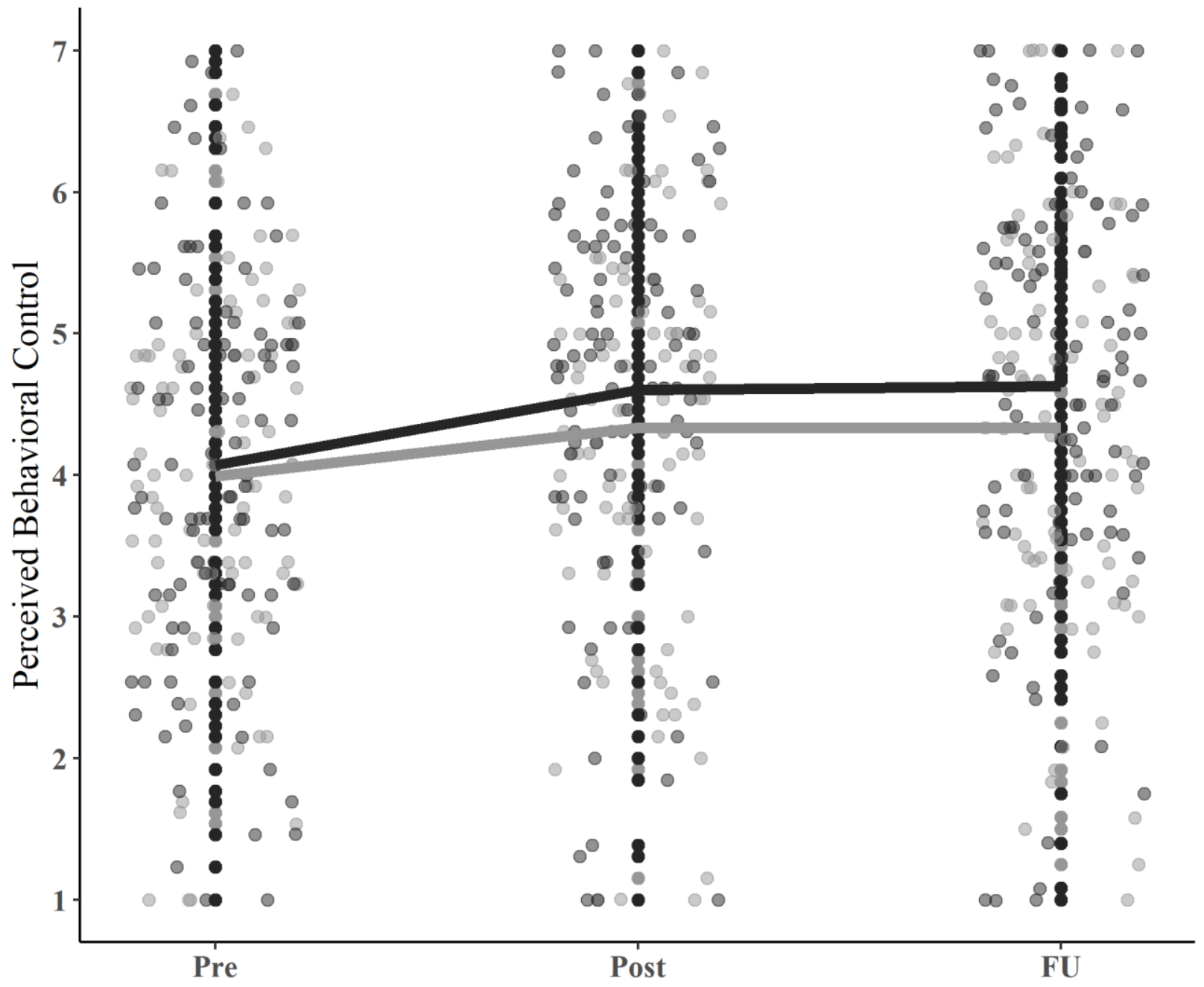

\section{Condition Education Motivation}

Note. Perceived behavioral control significantly improved from Pre to both Post and FU ( $p$ 's $<$ $.001)$, but there was no significant difference in this improvement between treatment conditions (p's $\geq .27$ ) (see Results). Perceived behavioral control was measured using a 13 item 1-7 Likert scale measure in which higher scores represent higher perceived behavioral control over seeking treatment for SAD (see Method). 